\title{
Recurrent Olfactory Groove Meningioma Filling the Nasal Cavity: A Case Report and Technical Video
}

\author{
Luciano Mastronardi ${ }^{1,2}$ Francesco Corrivetti ${ }^{1}$ \\ ${ }^{1}$ Division of Neurosurgery, Department of Surgical Specialties, San \\ Filippo Neri Hospital/ASL Roma1, Rome, Italy \\ ${ }^{2}$ Department of Neurosurgery, The State Education Institution of \\ Higher Professional Training The First Sechenov Moscow State \\ Medical University under Ministry of Health, Russian Federation \\ ${ }^{3}$ Division of ENT, Department of Surgical Specialties, San Filippo \\ Neri Hospital/ASL Roma1, Rome, Italy \\ ${ }^{4}$ Federal Centre of Neurosurgery, Tyumen, Russian Federation \\ Indian J Neurosurg 2021;10:159-161.
}

\author{
Pio Bevilacqua ${ }^{3}$ Raffaelino Roperto ${ }^{1}$ Albert Sufianov ${ }^{2,3}$
}

\author{
Address for correspondence Luciano Mastronardi, MD, PhD, \\ Division of Neurosurgery, Department of Surgical Specialties, San \\ Filippo Neri Hospital/ASL Roma1, Via Reno 14, Rome 00198, Italy \\ (e-mail: mastro@tin.it).
}

\begin{abstract}
Keywords

- Endoscopy

- meningioma

- sinonasal tract

- surgery

Background Meningiomas are usually benign tumors and account for $15 \%$ of the overall intracranial tumors. Less than $3 \%$ of them extend up to the sinonasal region. Case Report A 61-year-old woman, operated on for a huge olfactory meningioma 8 years before, came for progressive nasal obstruction. On physical examination, a mass that completely obliterated the right nasal passage was detected. A c.e. cranial MRI confirmed the presence of an enhancing mass completely occupying the right nostril, without intracranial extension. A biopsy of the mass revealed a meningioma (WHO grade I) and the patient was admitted to our department for the surgical removal of the endonasal recurrent tumor.

Results The meningioma was approached and excised by endonasal endoscopic approach using a microdebrider and electrocautery. A linear residual of tumor was left attached to the previous anterior skull base' vascularized galeal flap, in order to avoid a communication with the intracranial compartment and a possible cerebrospinal fluid (CSF) leak. The postoperative course was uneventful, and the patient immediately referred an improvement in breathing. A c.e. postoperative MRI confirmed the nearly total removal of tumor.

Conclusions We present a video with the most relevant steps of transnasal endoscopic surgical removal of a quite rare case of a recurrent olfactory groove meningioma, completely obliterating the right nostril.
\end{abstract}

\section{Introduction}

Meningiomas are tumors that usually have a benign character and a tendency to slow growth. ${ }^{1}$ They account for $15 \%$ of the overall intracranial tumors. ${ }^{2}$ They may cause various symptoms because of the compression of intracranial structures. Less than $3 \%$ of meningiomas may extend up to the sinonasal region. Meningioma is a common intracranial neoplasm with a variety of histomorphologic growth patterns which are usually easily recognized. However, primary extracranial meningiomas of the nasal cavity, paranasal sinuses, and nasopharynx (sinonasal tract) are rare..$^{3-5}$ We report a rare case of recurrent olfactory groove meningioma, arising from the anterior skull base and completely obliterating the right nostril in the light of the literature. published online

July 15, 2021
DOI https://doi.org/

$10.1055 / \mathrm{s}-0040-1716942$

ISSN 2277-954X. (c) 2021. Neurological Surgeons' Society of India.

This is an open access article published by Thieme under the terms of the Creative Commons Attribution-NonDerivative-NonCommercial-License, permitting copying and reproduction so long as the original work is given appropriate credit. Contents may not be used for commercial purposes, or adapted, remixed, transformed or built upon. (https://creativecommons.org/licenses/by-nc-nd/4.0/).

Thieme Medical and Scientific Publishers Pvt. Ltd. A-12, 2nd Floor, Sector 2, Noida-201301 UP, India 


\section{Case Report}

A 61-year-old woman, operated on for a huge olfactory meningiomas 8 years before, came for progressive nasal obstruction. On physical examination, a mass that completely obliterated the right nasal passage was detected. A c.e. cranial MRI confirmed the presence of an enhancing mass completely occupying the right nostril, without intracranial extension. A biopsy of the mass revealed a meningioma (WHO grade I), and the patient was admitted to our department for the surgical removal of the endonasal recurrent tumor.

The patient, therefore, was directed to our clinic. On her physical examination, a mass completely filling the left nasal passage and pushing the septum to the opposite side was observed. Findings of cranial MRI of the patient revealed a mass in the left nasal cavity with a size of $25 \times 34 \times 43 \mathrm{~mm}$, which appeared as a hypointense lesion on $\mathrm{T} 1$-weighted sequences, and as a heterogeneous and mildly hyperintense lesion on T2-weighted sequences, and showed homogeneous intense staining on the postcontrast examination, as well as a mass lesion in the bilateral maxillary sinus and the ethmoid sinus (Video 1). CT of the paranasal sinuses revealed a soft-tissue mass in the left nasal cavity, eroding the septum. The patient was hospitalized for surgical procedure.

\section{Video 1}

MRI revealed a mass in the left nasal cavity with a size of $25 \times 34 \times 43 \mathrm{~mm}$, which appeared as a hypointense lesion on T1-weighted sequences, and as a heterogeneous and mildly hyperintense lesion on T2-weighted sequences, and showed homogeneous intense staining on the postcontrast examination, as well as a mass lesion in the bilateral maxillary sinus and the ethmoid sinus. Online content including video sequences viewable at: https://www. thieme-connect. com/products/ejournals/html/ 10.1055/ s-0040-1716942

The operation was initiated with the endonasal endoscopic method.

The meningioma was approached and excised by endonasal endoscopic approach using a microdebrider and electrocautery. A linear residual of tumor was left attached to the previous anterior skull base' vascularized galeal flap, in order to avoid a communication with the intracranial compartment and a possible cerebrospinal fluid (CSF) leak. The postoperative course was uneventful, and the patient immediately referred an improvement in breathing. A c.e. postoperative MRI confirmed the nearly total removal of tumor. Trace of the mass revealed that it originated from the anterior skull base, and an approximately $5-\mathrm{mm}$ defect was detected in the skull base. The mass was excised by dissecting the skull base, including a part of the olfactory fibers and the posterior aspect of the septum. It was observed that the middle concha was extremely atrophied because of the compression effect of the mass. The defective area in the skull base was toured around by a diamond burr.
The middle concha was excised and placed onto the defect with the overlay technique. Polyps originating from the right middle concha were also excised using a microdebrider.

Histopathology of the mass in the left nasal passage was reported as meningothelial (syncytial) tumor composed of lobules of neoplastic cells with indistinct borders and round-to-oval nuclei with delicate chromatin (type 1 meningioma); the $\mathrm{K}_{\mathrm{i}}-67$ proliferation index was determined to be $1 \%$. The patient developed no postoperative complication.

\section{Discussion}

Meningiomas are generally benign tumors originating from the arachnoid cap cells. ${ }^{4}$ They are most commonly encountered after the fifth decade of life. The frequency of meningioma in females is two times higher than that in males. Meningiomas are often asymptomatic and detected incidentally. A small number of meningiomas may cause various symptoms including cranial nerve paralysis or seizure attacks according to their locations. Of the meningiomas, $20 \%$ extend up to the extracranial structures such as the calvarium, the orbita, and the ear. Less than $3 \%$ of intracranial meningiomas extend up to the nasal cavity. ${ }^{3}$ Long-term prognosis of meningiomas depends on histologic grade and resectability of the tumor.

Nowadays, meningiomas are histopathologically classified as benign (grade I), atypical (grade II), and malignant (grade III). Approximately $90 \%$ of meningiomas are benign, $5 \%$ are malignant, and 2 to $5 \%$ are atypical. ${ }^{1}$ Recently, $\mathrm{K}_{\mathrm{i}}-67$, which is a nuclear antigen found during cell proliferation, has been considered as a measure for grading the tumor and classifying the tumor as benign, malignant, or atypical. ${ }^{4}$ In the current study, meningioma was benign (grade I) and the $\mathrm{K}_{\mathrm{i}}-67$ proliferation index was $1 \%$.

CT and MRI are the most commonly used scanning methods for meningiomas. CT is useful in demonstrating bone destruction, whereas MRI is more beneficial in the evaluation of tumoral tissue. In MRI, meningiomas may appear as isointense or hypointense lesions on T1-weighted sequences, whereas they may be observed as isointense, heterogeneous, or hyperintense lesions on T2-weighted sequences. ${ }^{2}$ Cranial MRI of the current study revealed hypointense lesions on T1-weighted sequences and heterogeneous and mildly hyperintense lesions on T2-weighted sequences.

The primary treatment of meningiomas is complete surgical removal of the tumor. Endonasal endoscopic approach is a method that does not require cerebral retraction and yields good ophthalmological outcomes in the treatment of tumors, extending from the anterior skull base to the nasal cavity. ${ }^{6,7}$ Anterior skull base defects can be covered by a multilayer approach including collagen sponge matrix (Duragen, Tissufleece), Tutoplast grafts, resorbable solid material (Lactosorb, Lorenz Surgical), and fibrin glue (Baxter). Previous studies have demonstrated that the type of graft, overlay/ underlay technique, multilayer graft, and fixator (fibrin glue, Surgicel) have no significant difference in treatment success 
in covering the anterior skull base. ${ }^{8,9}$ The overlay technique is easier than the underlay technique. This technique requires precise removal of the mucosa around the defect to provide tight stabilization between the graft and the bone above the graft. The middle concha graft is ideal for this method. ${ }^{8}$

In the current study, the surgery was initiated with the endonasal endoscopic approach. The mass was excised up to the defect in the skull base using a microdebrider. Mucosal tissues around the defect were removed by a diamond burr. The middle concha was excised, shaped and then placed on the 5 -mm defect on the anterior skull base with the overlay technique. Two dissolvable nasal packs (Nasopore) were placed. The patient developed no postoperative complication, and no relapse was observed during the 2-year follow-up period.

\section{Funding}

None.

Conflict of Interest

None declared.

\section{References}

1 Jalisi S. Atypical meningioma presenting as a nasal massmultidisciplinary management. J Craniomaxillofac Surg 2012;40(4):e115-e118
2 Mnejja M, Hammami B, Bougacha L, et al. Primary sinonasal meningioma. Eur Ann Otorhinolaryngol Head Neck Dis 2012;129(1):47-50

3 Das S, Das D, Ashraf K, Prinsley P. An unusual nasal polyp: skull base meningioma with extracranial extension into the nasal cavity. Br J Hosp Med (Lond) 2012;73(1):46-47

4 Commins DL, Atkinson RD, Burnett ME. Review of meningioma histopathology. Neurosurg Focus 2007;23(4):E3

5 Lindström CG, Lindström DW. On extracranial meningioma. Case of primary meningioma of nasal cavity. Acta Otolaryngol 1969;68(5):451-456

6 Fernandez-Miranda JC, Gardner PA, Prevedello DM, Kassam AB. Expanded endonasal approach for olfactory groove meningioma. Acta Neurochir (Wien) 2009;151(3):287-288, author reply 289-290

7 de Divitiis E, Esposito F, Cappabianca P, Cavallo LM, de Divitiis O, Esposito I. Endoscopic transnasal resection of anterior cranial fossa meningiomas. Neurosurg Focus 2008; 25(6):E8

8 Di Rocco F, Couloigner V, Dastoli P, Sainte-Rose C, Zerah M, Roger G. Treatment of anterior skull base defects by a transnasal endoscopic approach in children. J Neurosurg Pediatr 2010;6(5):459-463

9 Banks CA, Palmer JN, Chiu AG, O'Malley BW Jr, Woodworth BA, Kennedy DW. Endoscopic closure of CSF rhinorrhea: 193 cases over 21 years. Otolaryngol Head Neck Surg 2009; 140(6):826-833 